\title{
Metastatic Signet Ring Cell Adenocarcinoma-An Autopsy Finding in Myocardium: A Rare Case Report
}

\author{
${ }^{1}$ Department of Pathology, All India Institute Of Medical Science, \\ Bathinda, Punjab, India \\ 2Department of Pathology, Government Medical College \& Hospital, \\ Chandigarh, India \\ ${ }^{3}$ Department of Forensic Medicine, Government Medical College \& \\ Hospital, Chandigarh, India
}

Jasmeen Kaur ${ }^{1}$ Amarvir Kaur ${ }^{2}$ Rajpal Singh Punia ${ }^{2}$ Harish Dsari ${ }^{3}$

\begin{abstract}
Address for correspondence Jasmeen Kaur, MD, DNB, Department of Pathology, All India Institute Of Medical Science, Bathinda 151001, Punjab, India (e-mail: jasmeenduggal66@gmail.com).
\end{abstract}

Asian J Oncol 2021;7:149-151.

\begin{abstract}
Introduction Stomach is the most common primary site of signet ring cell adenocarcinoma unlike breast or pancreas. The distant metastasis is commonly seen in peritoneum, liver, and lungs. Few cases have been reported on metastasis in pericardial fluid with presentation of cardiac tamponade. However, to the best of my knowledge, myocardium is one of the rarest sites of distant metastasis.

Case Report A 26-year-old male had abdominal pain at home and was brought dead to the hospital. The postmortem examination was done, and heart was sent for histopathological report. The gross examination of heart, lungs, liver, kidneys, and spleen were unremarkable and on microscopy, sections examined from lung and left ventricular wall showed infiltration by a tumor composed of signet ring cells and few glands in the background of the mucinous material and the primary site of tumor was suggested

Keywords

- autopsy

- myocardium

- lungs

- signet ring cell

carcinoma to be stomach and rectum, whereas sections examined from liver, kidneys and spleen did not show any significant pathological change or any metastatic deposit.

Conclusion Distant metastasis of signet ring cell carcinoma is mostly seen in peritoneum, liver, and lungs. So, to conclude myocardium is the rarest site of distant metastasis. Therefore, the heart autopsy is of utmost importance to look for the cause of death and also important from the perspective of academic value.
\end{abstract}

\section{Introduction}

Gastrointestinal tract is the most common site of signet ring cell carcinoma; however, it can arise from any organ. ${ }^{1}$ In $90 \%$ of the cases, stomach is the most common site. It has high risk of metastasis as it invades the submucosa, then serosa. It appears at an advanced stage IV because of its feature of late presentation. ${ }^{2}$ It spreads to various sites including serosal surfaces, retroperitoneum, gastric mucosa, and ovaries. Many case reports showed metastatic deposits at various other sites including the rare ones like cardiac tamponade, pericardial fluid, and peritoneal cavity. ${ }^{3}$ However, to the best of my knowledge, there is no case report on metastatic deposit of

published online

December 30, 2020
DOI https://doi.org/

10.1055/s-0040-1722109 ISSN 2454-6798. signet ring cell carcinoma in myocardium. Moreover, autopsy finding of metastatic deposit of signet ring cell carcinoma in myocardium has never been reported before.

\section{Case Report}

A 26-year-old male complained of pain in abdomen at home for 6 to 12 hours and brought dead at GMCH-32. A detailed postmortem examination was done in forensic department. The postmortem examination showed that the deceased was average built with $162 \mathrm{~cm}$ height. Organs were sent for histopathology report. Organs including heart, portion of both lungs, portion of liver, portion of spleen, and portion of 
both kidneys, and portion of brain of suspected sites were sent to the Department of Pathology. Clinical postmortem findings were noted; therefore, postmortem report and police papers were sent along with viscera. Viscera was received in $10 \%$ buffered formalin solution. On gross examination, uncut heart was $220 \mathrm{~g}$ in weight and measured $10 \times 6 \times 5 \mathrm{~cm}$; the valve circumference of tricuspid, mitral, pulmonary, and aortic valve measured $11 \times 10 \times 8 \times 7 \mathrm{~cm}$, respectively. The left and right ventricular wall thickness measured 1.5 and $0.4 \mathrm{~cm}$. The coronaries were traced are far as possible and were found to be patent ( $\boldsymbol{- \text { Fig. }}$ 1). Also, the portion of right lung measured $7 \times 5 \times 1 \mathrm{~cm}$ and weighed $60 \mathrm{~g}$ and left lung measured $4 \times 3 \times 1 \mathrm{~cm}$ with a weight of $30 \mathrm{~g}$. Externally unremarkable whereas gross examination of liver, spleen, and both kidneys and brain was unremarkable. Multiple sections were taken from all proposed areas and abnormal looking areas. After routine tissue processing, hematoxylin and eosin staining was done. On microscopy, sections examined from left ventricular wall showed infiltration of the myometrium by a tumor composed of signet ring cells and few glands in the background of the mucinous material. The tumor was seen on the pericardium as well ( - Figs. 2 and 3). On microscopy from both lungs, the alveoli showed infiltration by a tumor composed of signet ring cells and few glands in the background of the mucinous material along with the presence of eosinophilic proteinaceous material and congested blood vessels in the intra-alveolar septa ( - Figs. 4 and $\mathbf{5}$ ). Also, microscopic examination of liver, spleen, both

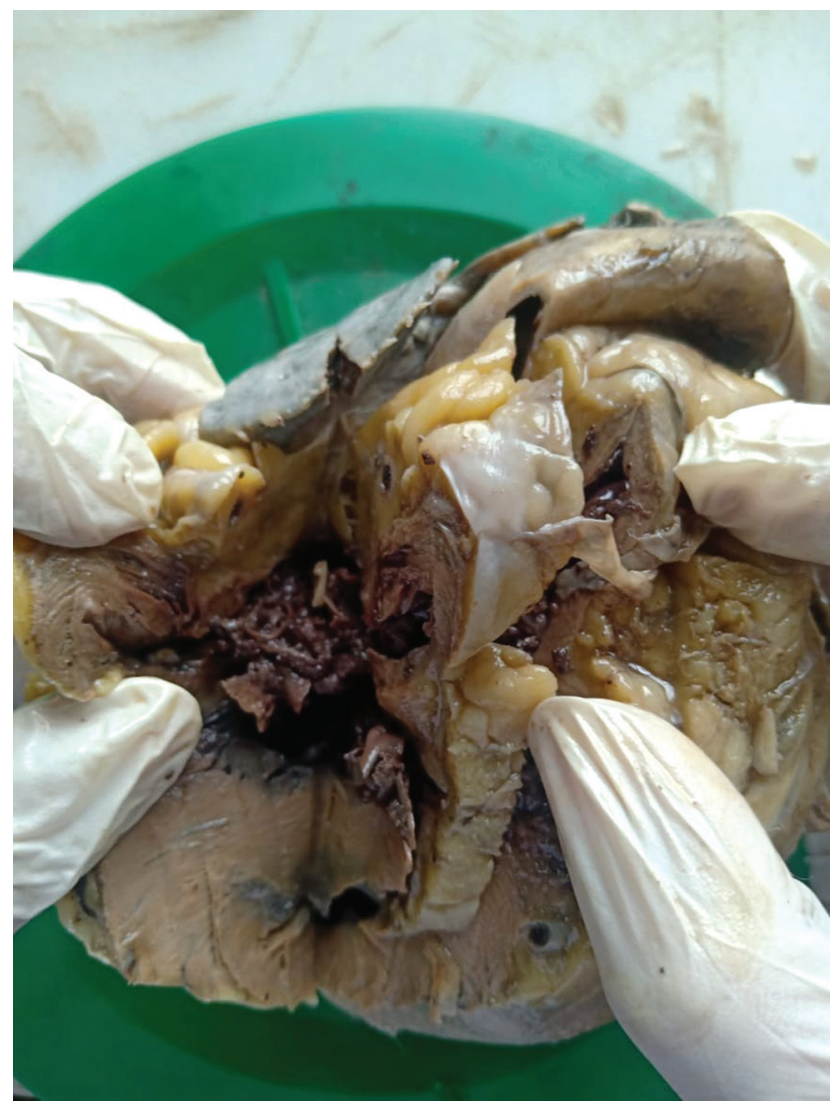

Fig. 1 Gross picture of heart. kidneys, and brain did not show any significant pathological change. The final diagnosis was given as the metastatic signet ring cell adenocarcinoma and the most common possible site for primary includes stomach and rectum.

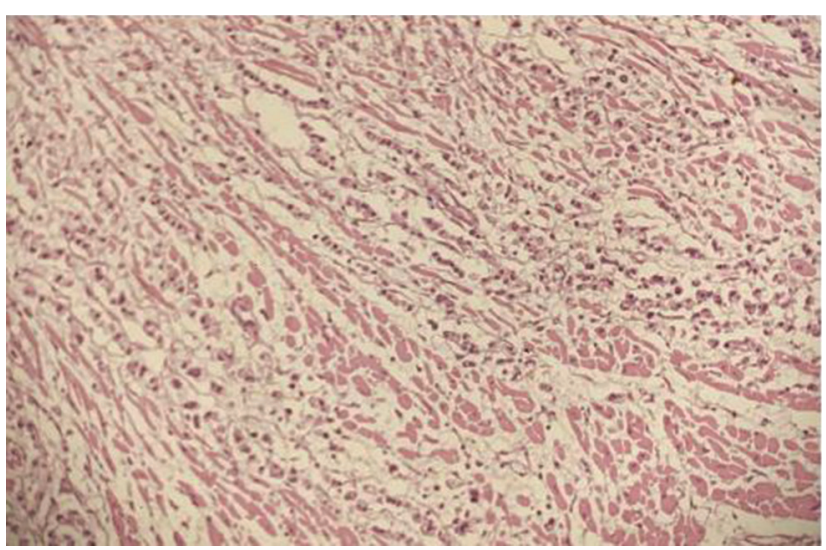

Fig. 2 H\&E section from left ventricular wall showing metastatic deposits of signet ring cell carcinoma (200x). H\&E, hematoxylin and eosin staining.

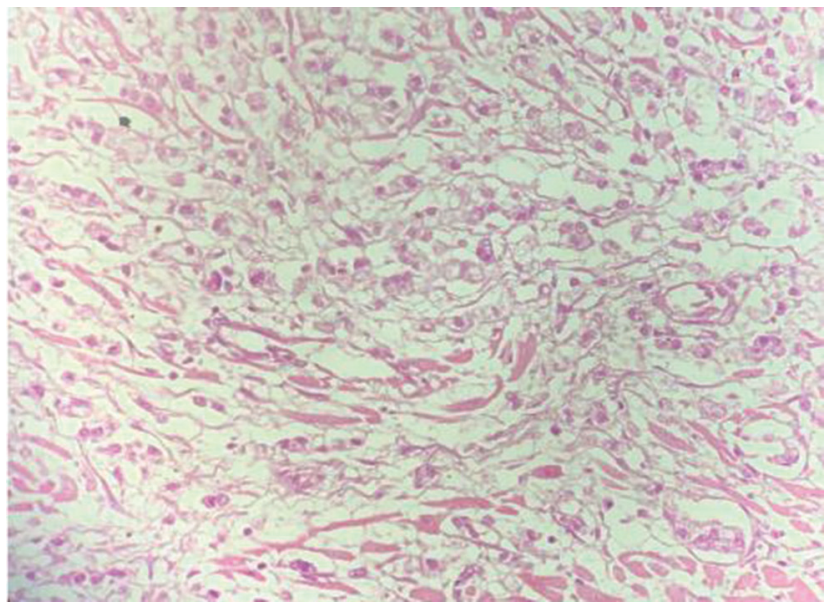

Fig. 3 H\&E section from left ventricular wall shows metastatic deposits of signet ring cell carcinoma (400×). H\&E, hematoxylin and eosin staining.

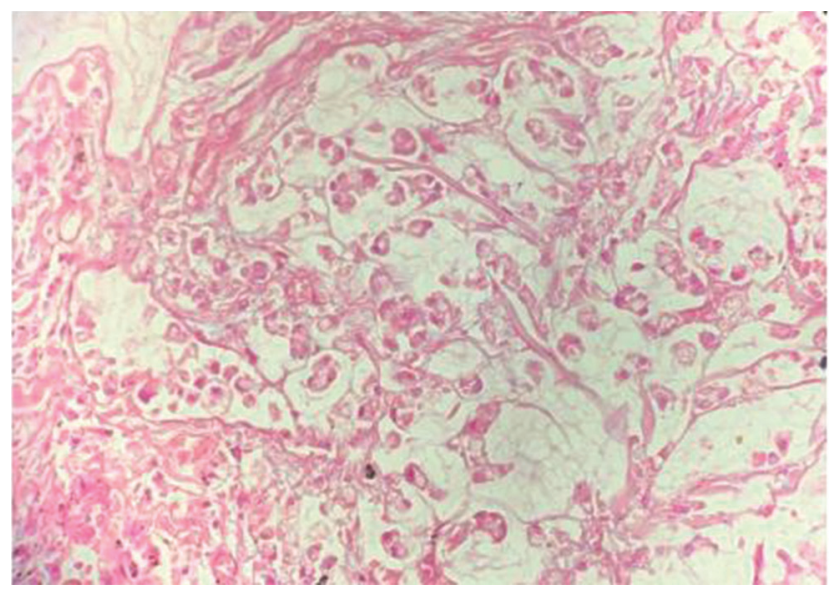

Fig. $4 \mathrm{H} \& \mathrm{E}$ sections from lung showing signet ring cells at high power (400X). H\&E, hematoxylin and eosin staining. 


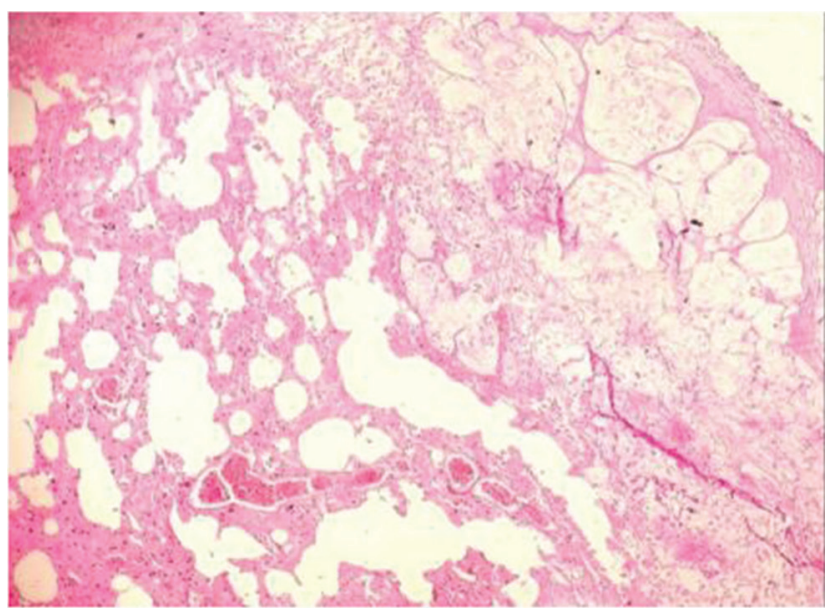

Fig. $5 \mathrm{H} \& \mathrm{E}$ sections from lung showing metastatic deposit of signet ring cell carcinoma (200X). H\&E, hematoxylin and eosin staining.

\section{Discussion}

Primary signet ring cell is most commonly found in stomach; however, other sites of primary signet ring cell have also been reported like colon, pancreas, and breast and have extremely poor prognosis. It is usually seen in young adults. Various types of tumors have been described, like linitis plastica, when primary site is stomach ${ }^{3}$; if colon is the primary site it presents as scirrhous or ulcerated lesion. It is usually spread by lymphatic route and also synchronous with peritoneal and liver dissemination. Mostly patients present with chief complaints of abdominal pain or bleeding per rectum. Tumor has high rate of metastasis. The main routes by which the cardiac metastases commonly occur from primary tumors are: (1) by direct extension; (2) by lymphatic spread; (3) by hematogenous spread; and (4) by combinations. ${ }^{4}$ It clinically presents at stage IV. ${ }^{5}$ Some patients presented with cardiac tamponade. Pericardial biopsy showed metastasis by signet ring cell adenocarcinoma; on further investigation, the primary site was found to be the stomach. Distant metastasis is seen in the pericardial fluid and pericardium in few cases. ${ }^{6}$ However, in this case patient was having acute abdominal pain at home for 6 to 12 hours. The patient was taken to the local hospital and further referred to GMCH-32 where patient was declared brought dead. The autopsy was done to know the cause of sudden death. Histopathological examination of heart and lungs showed metastasis from signet ring cell carcinoma and primary tumor being suggested to be in stomach or rectum. A MEDLINE database search of the showed case report of primary gastric carcinoma presenting as cardiac tissue infiltration was reported in the literature between 1982 and 2010. Confirmation was done on postmortem examination which correlated with the presenting symptoms of cardiac tamponade. The case has been reported as a primary signet ring cell carcinoma of the stomach. ${ }^{3}$ Therefore, the treating clinicians should consider pericardial disease in any patient with or without a known malignancy who present with clinical features of unexplained breathlessness, or symptoms of heart failure. ${ }^{7}$ Varvarigos et $a{ }^{8}{ }^{8}$ elaborated the presence of clinical features of cardiac tamponade as the first sign of malignancy in stomach in some patients ( - Fig. $\mathbf{3}$ ).

\section{Conclusion}

The primary site of signet ring cell adenocarcinoma is most commonly the stomach, and breast, pancreas, etc. are less common. Distant metastasis is commonly found in peritoneum, lungs, and liver. To the best of my knowledge, no case has been rWeported in myocardium, as the prognosis of signet ring carcinoma is extremely poor because of its late presentation. Therefore, it showed that primary gastric carcinoma can present as cardiac tamponade which is rare and physician should have knowledge of malignancy of the stomach when patients present with unexplained cardiac manifestations. As highlighted, it is a rare presentation, so to make correct diagnosis and to find the cause of death, heart autopsies are of utmost importance and also of academic value.

Conflict of Interest

None declared.

\section{References}

1 Dayapala A, Murty OP. Operational guidelines for postmortem examinations. J Forensic Med Toxicol 2006;23(1):9-43

2 Belli S, Aytac HO, Karagulle E, Yabanoglu H, Kayaselcuk F, Yildirim S. Outcomes of surgical treatment of primary signet ring cell carcinoma of the colon and rectum: 22 cases reviewed with literature. Int Surg 2014;99(6):691-698

3 Huang J-Y, Jiang H-P, Chen D, Tang H-L. Primary gastric signet ring cell carcinoma presenting as cardiac tamponade. World J Gastrointest Oncol 2011;3(4):67-70

4 MoriyamaA,MurataI,KurodaT, etal.Pericardiacmetastasisfrom advanced gastric cancer. J Gastroenterol 1995;30(4):512-516

5 Makino T, Tsujinaka T, Mishima H, et al. Primary signet-ring cell carcinoma of the colon and rectum: report of eight cases and review of 154 Japanese cases. Hepatogastroenterology 2006;53(72):845-849

6 Hashimoto Y, Iwata Y, Sangen R, et al. Pericardial biopsy revealed gastric signet-ring cell cancer. Case Rep Oncol 2015; $8(1): 174-178$

7 Wilkes JD, Fidias P, Vaickus L, Perez RP. Malignancy-related pericardial effusion. 127 cases from the Roswell Park Cancer Institute. Cancer 1995;76(8):1377-1387

8 Varvarigos N, Kamaradou H, Kourti A, et al. Cardiac tamponade as the first manifestation of gastric cancer and remission after chemotherapy. Dig Dis Sci 2001;46(11):2333-2335 